\title{
Analysis of Traffic Performance of a Merging Assistant Strategy Using Cooperative Vehicles
}

\author{
Riccardo Scarinci ${ }^{1}$, Benjamin Heydecker ${ }^{1}$, Andreas Hegyi ${ }^{2}$
}

\begin{abstract}
Emerging in-car communication technologies continually offer new communication capabilities between vehicles and infrastructure that, together with more accurate positioning systems, can be used to improve the use of current infrastructure. The aim of this paper is to present a novel merging assistant strategy that exploits cooperative systems to reduce congestion at motorway junctions. This new system, called Cooperative Merging Assistant, groups main carriageway vehicles together and collects the inter-vehicle spaces into gaps that are usable by merging traffic. These gaps will facilitate the coordinated entry of platoons of vehicles released by an on-ramp traffic signal. The performance of this new system is evaluated using microscopic simulation. Results show the reduction of late-merging vehicles, decrease in congestion and increase of merging capacity. This study shows how the use of cooperative systems can improve the the merging maneuver and so lead to a reduction of congestion on motorways.
\end{abstract}

\section{INTRODUCTION}

Motorway traffic congestion is a phenomenon experienced by millions of drivers every day with significant economic losses. Nowadays, new capabilities offered by emerging in-car communication technologies enabling communication between vehicles (V) and infrastructure (I), more accurate positioning systems and more precise algorithms for data fusion and systems integration can be used to improve the use of infrastructure. These technologies, systems and algorithms offer new opportunities for cooperation among vehicles.

Since traffic flow theory has been developed, the nature of congestion and its principal causes have been discussed intensively. Recurrent congestion on motorways occurs at the same locations due to specific infrastructure features such as on-ramps, lane-drops, sharp bends; but it does not always happen under the same traffic conditions [1]. This empirical phenomenon is not described by classical traffic flow theory [2], according to which, the transition from free-flow to congested-flow, i.e. traffic flow breakdown, occurs only when the density of the vehicles exceeds a critical value. Prigogine and Herman suggested that breakdown does not occur in this deterministic way but has a stochastic nature [3]. If the traffic density is less than the critical value, then breakdown happens at a certain probability per unit time that increases with traffic density. This concept of rate of breakdown is related to that of stochastic motorway capacity as introduced and empirically analysed by Brilon et al. [4]. While [3] and [4] proposed a stochastic nature of breakdown and capacity,

\footnotetext{
${ }^{1}$ Centre for Transport Studies, Dep. of Civil, Environmental \& Geomatic Engineering, University College London (riccardo.scarinci.09@ucl.ac.uk, b.heydecker@ucl.ac.uk)

${ }^{2}$ Dep. of Transport \& Planning, Fac. of Civil Engineering \& Geosciences, Delft University of Technology (a.hegyi@ tudelft.nl)
}

other authors identified the perturbations of merging vehicles as the cause of breakdown at on-ramp merges [5], [6]. These perturbations can be created by aggressive driving, relaxation phenomenon and by vehicles that are not able to find a suitable gap during the merging manoeuvre [7]; therefore, they are forced to decrease their speed when they approach the end of the acceleration lane. These latemerging vehicles will then accept smaller gaps and merge with lower speeds, thus disrupting the main carriageway vehicles. This phenomenon can trigger a transition from freeflow to congested-flow even if the vehicle density is lower that the critical one. Leclercq et al. described the behaviour of the merging vehicles as moving bottlenecks responsible for a capacity drop at the merging sections, identifying the difference in speed between the merging vehicles and the main carriageway traffic as the principal cause [8].

The most widespread traditional ITS system for managing motorway junctions is ramp metering (RM). RM aims to regulate vehicles entering the motorway from the on-ramp to avoid congestion on the main carriageway. In the last 40 years, different control strategies have been developed for this purpose [9], for example: demand-capacity strategy based on downstream occupancy and upstream flow [10]; ALINEA based on downstream occupancy and a closed loop control strategy [11]; ANCONA based on upstream speed [12]; and strategies based on a model predictive control approach [13]. All these control strategies are based on traditional technologies, such as detector loops and traffic lights, to prevent congestion, and in particular they do not make use of cooperation from main carriageway vehicles to reduce disruptions caused by late-merging vehicles. On the other hand, innovative traffic management strategies can exploit the capabilities offered by emerging communication technologies to request cooperation between on-ramp and main carriageway vehicles [14]. Early work on managed vehicle merging focused on the safety of the merging manoeuvre rather than improving traffic flow and avoiding congestion [15]-[17]. Subsequently, Davis studied the use of Cooperative Adaptive Cruise Control systems, capable of detecting vehicles on the on-ramp, and facilitating the merging of individual vehicles improving motorway throughput [18]. Daamen et al. investigated a similar approach, focusing the analysis on the effects of sending messages to individual vehicles as soon as its behaviour could disturb the traffic flow, eg a main carriageway vehicle hindering merging vehicle at on-ramp [19]. While the control strategies developed in [18] and [19] modify the speed of main carriageway vehicles, Wang et al. presented a proactive traffic control algorithm 
for facilitating the merging of equipped cars which modified the speed of on-ramp vehicles [20]. This algorithm identifies the most suitable gap on the main carriageway and suggests an appropriate speed for the merging vehicle well before it arrives at the merging point. More recently, Pueboobpaphan et al. studied again the effects of Cooperative Adaptive Cruise Control for merging assistance in case of mixed traffic, also considering the effects on string stability [21]. Park et al., instead of reducing the speed of main carriageway vehicles to create gaps, proposed an algorithm providing lane changing advice to motorway main carriageway vehicles to create larger gaps for merging vehicles [22]. Finally, Marinescu et al. suggested a merging algorithm with cooperation between main carriageway vehicles and on-ramp vehicles based on a slot-based traffic management system [23]. All the presented works facilitate the merging of on-ramp vehicles using innovative communication technologies, but none of them integrates these technologies with the already present ramp metering infrastructure.

The aim of this study is to present a novel control strategy for cooperative merging and to evaluate its effects on traffic flow. This new system, called Cooperative Merging Assistant (CoopMA), rearranges gaps present in the motorway main carriageway by requesting cooperation from participating vehicles in order to facilitate the merging of on-ramp platoons. These platoons are released by a traffic light already present in junctions equipped with traditional ramp metering, where, for operational or legislative reasons, a Full Traffic Cycle metering policy is necessary, i.e. a single platoon is released at each traffic light cycle [24]. This new control strategy exploits the opportunity offered by the integration between intelligent vehicles equipped with on-board communication systems and ramp metering technology, investigating possible benefits of the enabled cooperation among vehicles. This control strategy, creating suitable gaps for merging, should be able to reduce the disruption of on-ramp vehicles and therefore reduce the occurrence of congestion at junctions [25].

\section{Cooperative Merging Assistant control STRATEGY}

This section presents a new control strategy and explores its spatio-temporal effects on traffic flow and vehicle density, as well as introducing the methodology used to define the control algorithm.

To facilitate merging, the proposed control strategy coordinates the release of platoons of on-ramp vehicles with the gaps created on the main carriageway specifically for them. These gaps are created by collecting those already present on the near-side lane, i.e. the lane closest to the on-ramp, and compacting the vehicles into groups of a higher density. This rearrangement can be achieved by reducing the speed of one vehicle on the main carriageway equipped with an incar communication system. The speed of this vehicle, called cooperative vehicle, can be reduced either automatically, transmitting instructions to the vehicle Cruise Control, or

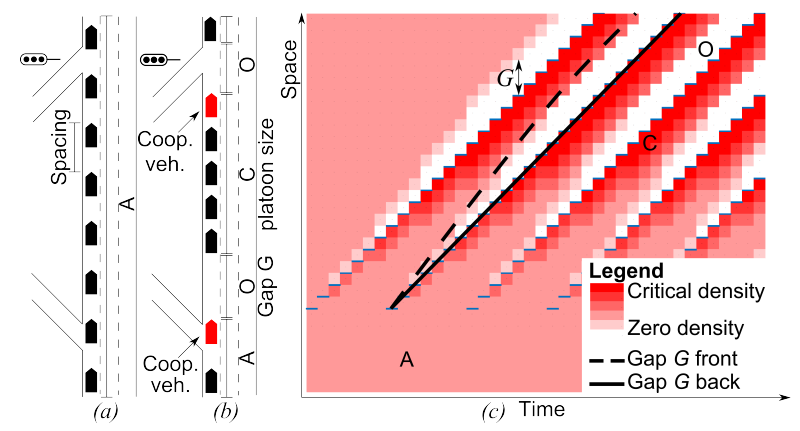

Fig. 1. Conceptual representation of the effects on traffic flow under Cooperative Merging Assistant. (a) vehicle configuration if CoopMA is not applied, and $(b)$ if CoopMA is applied with creation of platoons and gaps. (c) representation of the spatio-temporal evolution of the main carriageway vehicle density with formation and evolution of gaps $G$ suitable for merging created by the decrease in speed of the cooperative vehicles.

manually, showing a message on an on-board display and requesting a lower speed to be maintained.

Figures $1(a)$ and $(b)$ show the difference in vehicle configuration on the main carriageway if this system is not applied (a) or if it is applied (b). Figure 1(c) shows a conceptual representation of the spatio-temporal diagram of the main carriageway vehicle density if CoopMA is applied. Once the cooperative vehicle reduces its speed, the gaps between vehicles are rearranged; and a gap $G$ is gradually expanded in front of the cooperative vehicle. Meanwhile, the upstream vehicles, due to the slow vehicle in front, will compact behind the cooperative vehicle, expanding the area of high density. If, subsequently, another cooperative vehicle is set, this effect will be re-created cyclically. This gap is represented in Figure 1(b) as the distance between the front of the cooperative vehicle and the rear of the next vehicle in front, and as an area of zero density in Figure 1(c). The space-time evolution of the upstream and downstream fronts of the gap $G$ is represented in Figure 1(c). The upstream front of the gap is determined by the cooperative vehicle trajectory in the spatio-temporal diagram. The evolution of the downstream front is determined by the trajectory of the first vehicle downstream from the cooperative vehicle, i.e. the last vehicle in the platoon between two consecutive cooperative vehicles (the back of the platoon).

In order to develop the Cooperative Merging Assistant control strategies, the evolution of the gap is calculated analytically. Because the focus of the present paper is on the traffic performance of the CoopMA system rather than the definition of the control strategy, only a brief explanation is presented of the methodology. A complete discussion of the methodology, the equations and the results on the CoopMA control strategy can be found in [25], [26].

The fundamental diagram of traffic flow, its properties and shock wave theory have been used to define the CoopMA control strategy analytically. The fundamental diagram was used to calculate the size of the gap that it is possible to create for different main carriageway traffic conditions and platoon sizes. Every traffic state $\phi$ is defined by its speed $v_{\phi}$, density $k_{\phi}$ and flow $q_{\phi}$, and the fundamental diagram defines 

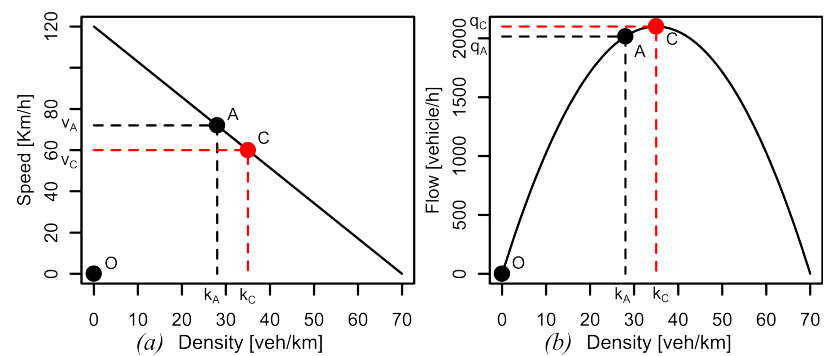

Fig. 2. Conceptual speed-density ( $a$ ) and flow-density (b) diagrams with the representation of the different traffic state created by the use of Cooperative Merging Assistant.

the relationships among these variables. Figure 2 shows a conceptual parabolic fundamental diagram that is used here for describing the CoopMA control strategy. Assuming that the actual traffic state upstream of the on-ramp is known (measured) to be $A$, using the fundamental diagram, it is possible to determine each of its speed $v_{A}$, density $k_{A}$ and flow $q_{A}$, identified by point $A$ in Figure 2 . If the cooperative vehicle slows down to speed $v_{C}$, after a certain amount of time, the vehicles immediately upstream will travel at a higher density $k_{C}$, shown as state $C$ in Figure 2 . The spacing between the vehicles is reduced; so, if a further cooperative vehicle is set, a gap is created between the last vehicle in the platoon and the next cooperative vehicle, corresponding to state $O$ (origin state). The speed $v_{C}$ of the cooperative vehicle is a control strategy design variable and should be chosen to lie in the range from $v_{A}$ to the speed that maximises flow, i.e. the critical speed. Note that state $A$ is stable by definition, and state $C$ has to be chosen such that it is also stable [25]. This will ensure that no breakdown is caused by slowing down the cooperative vehicles. Empirical data in proximity of the on-ramp to manage can be used to identify these states. The size, in time or space, of the gap is a function of state $A$ (measured), state $C$ (design variable) and the number of vehicles between two consecutive cooperative vehicles (design variable), i.e. the platoon size as shown in Figure $1(b)$.

Meanwhile the fundamental diagram has been used to estimate the size of the gap, shock wave theory [2] has been used to determine the time and space required to create the gap, i.e. the spatio-temporal evolution of the boundaries between the traffic states $A, C$ and $O$, as shown in Figure 1(c). Shock wave theory relates the spatio-temporal diagram of the traffic states and the flow-density diagram. The latter diagram defines the traffic states $A, C$ and $O$, and the spatiotemporal diagram describes the evolution of the boundaries of these states in space and time. According to shock wave theory, the slope of the trajectory, i.e. the speed, of the boundary between two traffic states in the spatio-temporal diagram is equal to the slope of the line connecting the states on the flow-density diagram. Combining the trajectories of the boundaries with those of the vehicles it is possible to determine the time and space that the vehicles in the platoon will need to compact and to create the gap $G$, i.e. change from state $A$ to state $C$ and $O$. These quantities are of fun- damental importance, because they define when and where to send a message to the cooperative vehicle requesting the deceleration. The location of cooperation varies according to the traffic state on the main carriageway and the platoon size chosen; therefore it must be calculated dynamically [25], [26].

In order to calculate the traffic light phases durations for coordinating the release of the on-ramp vehicles with the gap $G$, beside the spatio-temporal evolution of the gap, the travel time from the traffic light on-ramp stop line to the merge location should be known. This can be estimated using kinematic equations with the average vehicle acceleration and the junction geometry. Now, the green and red phases can be defined as follow. The start of the green phase is triggered by the position of the cooperative vehicle communicated to the infrastructure. The green phase will begin when the predicted arrival at the merging location of the gap $G$ will match the predicted arrival of the on-ramp vehicles released by the traffic light. This is calculated to synchronise the arrival at the merging location of the first released on-ramp vehicle with the downstream front of the gap $G$. The duration of the green phase, and thus the number of vehicles in the platoon released in each traffic light cycle, is instead defined by the duration of the gap $G$. Supposing each on-ramp vehicle needs a fixed time gap to merge safely into the main carriageway, the duration of the green phase is calculated so that the number of vehicles released is the maximum able to merge in the gap $G$. Traditional RM installations calculate that a vehicle needs a fixed time of 2 second to cross the stopping line during the green phase [24], therefore the green phase is calculated accordingly. Finally, the length of the red phase is determined, such that in the next green phase, the on-ramp vehicles will be released to the gap that is created by the following cooperative vehicle. The total cycle length depends on the flow on the main carriageway, and the platoon size [25], [26].

The exchange of information requested by the CoopMA system is of two types:

(i) Vehicle to Infrastructure. On-ramp and main carriageway vehicles give information on the traffic state to the control centre. This communication can be done using the detector loops that are already present in junctions equipped with traditional RM. In addition, the position of the cooperative vehicles on the main carriageway is communicated to the control centre.

(ii) Infrastructure to Vehicle. The control centre releases the on-ramp vehicles and slows down cyclically the cooperative vehicles. For the release of on-ramp vehicles, the CoopMA uses a traffic signal that is already present in junctions equipped with RM, and so, no intelligent vehicles are required. On the other hand, for creating the main carriageway gap $G$, vehicles equipped with on board communication system are necessary.

The CoopMA strategy is designed to release platoons of vehicles instead of single vehicles for two main reasons. First, a moderate amount of V2I communication is required, given that only one intelligent vehicle for traffic light cycle 
is necessary. Second, coordinating a single large gap with a single platoon presents fewer difficulties than coordinating many small gaps for individual vehicles. This because the conflicts between the merging and main carriageway vehicles occur at the extremes of the merging platoon and the main carriageway gap $G$. Therefore, larger platoons have less conflicting zones per vehicle. The disadvantage of releasing platoons is that they create stronger disruptions on the main carriageway flow while merging [6], [24]. The effects of this design on the control strategy performance are evaluated using simulation in the next sections, while the consequences of miss-coordination between gaps and merging platoons are left for further work.

\section{EVALUATION METHODOLOGY}

To evaluate the effects of the Cooperative Merging Assistant system, traffic performance has been assessed for different scenarios on a motorway junction using a microscopic simulation. This evaluation aims to prove that the CoopMA system, by creating suitable gaps for merging, is able to reduce disruption caused by on-ramp vehicles and thus to reduce the occurrence of congestion at junctions.

The general research question of this evaluation is on the capability of the Cooperative Merging Assistant to improve traffic performance. Considering the control actions implemented by the CoopMA system for managing the motorway junction, this general research question can be split into four specific ones:

Q.1 Does the Cooperative Merging Assistant system reduce the occurrence of congestion at merges?

Q.2 Does the Cooperative Merging Assistant system reduce the congestion duration?

Q.3 Does the Cooperative Merging Assistant system reduce the number of late-merging vehicles?

Q.4 Does the Cooperative Merging Assistant system reduce the merging position?

To answer these questions, three scenarios have been chosen for the simulation: Reference - single entry uncontrolled; Without CoopMA - platoon entry without Cooperative Merging Assistant strategy; and CoopMA - the same platoon entry but controlled by Cooperative Merging Assistant strategy. The Reference scenario shows the traffic flow behaviour for the uncontrolled situation. The traffic light is not active (set to a permanent green), and the merging vehicles follow their individually determined trajectories merging one at the time. The Without CoopMA scenario simulates the merging of a platoon released by a traffic light without any cooperation from the main carriageway vehicles. In order to have a comparison among the scenarios, the traffic light phases match the one calculated by the CoopMA control strategy presented in the following; thus, the same platoon size is released. This scenario aims to provide a comparison between the effects of the same platoon of vehicles merging without and with the use of the CoopMA strategy. In the third scenario, the traffic light is controlled by the CoopMA control strategy and the main carriageway vehicles are cooperating for facilitating the merging.
The two design variables to be defined for the CoopMA strategy are: $\Delta v$ the maximum reduction in speed of the cooperative vehicles, and $n_{\mathrm{p}}$ the main carriageway platoon size. For these simulations the values $\Delta v=10 \mathrm{~km} / \mathrm{h}$ and $n_{\mathrm{p}}=10$ vehicles have been chosen. A cooperative vehicle is assumed to be available each time one is required, therefore the distribution of cooperative vehicles is exactly 1 followed by 9 normal vehicles. The traffic light phases and the cooperation location, defined by the CoopMA control strategy, are constant during the entire simulation, because the main carriageway flow, i.e. state $A$, and the platoon size are constant. The traffic light cycle time is of 18.0 seconds with a green phase duration of 7.5 seconds, sufficient to allow the maximum simulated on-ramp flow of $900 \mathrm{veh} / \mathrm{h}$, followed by a red phase of 10.5 seconds. The cooperative vehicle should start to decrease its speed and to create the gap $G$ at least $1.8 \mathrm{~km}$ upstream of the merging location, according to the calculations discussed in Section II.

Beside the stated differences, the scenarios have other common characteristics. All simulations have a duration of 30 minutes, chosen as a trade-off between being representative of a peak hour and computational time. To simplify the simulation and gain a clearer evaluation of the control effect, a small variability in the vehicle characteristics has been introduced. All the vehicles have identical parameters except for their desired speed, which is generated from a truncated normal distribution with mean $v_{o}=120 \mathrm{~km} / \mathrm{h}$, standard deviation $1 \mathrm{~km} / \mathrm{h}$ and cut at $\pm 2 \mathrm{~km} / \mathrm{h}$. Once again to simplify the simulations, the vehicles have been generated with a constant headway, instead of the default Poisson process used by the simulation package. However, given the $4 \mathrm{~km}$ length of the upstream link and the different desired speeds, vehicles have time to cluster together after being generated, arriving at the merging location with a realistic distribution, as it will be shown in Section IV.

Each scenario has been evaluated over a range from absence of congestion to fully congested. The main carriageway flow was kept constant at $2,000 \mathrm{veh} / \mathrm{h}$, and the on-ramp demand was varied from $200 \mathrm{veh} / \mathrm{h}$ to $900 \mathrm{veh} / \mathrm{h}$, with increment of $50 \mathrm{veh} / \mathrm{h}$, for a total of 15 different onramp flows. Each flow was simulated for 30 runs each with a different random seed, and the distribution of the performance indexes has been used for comparison among scenarios instead of single values.

The simulated infrastructure, equal for the three scenarios, is composed of a single merge since the Cooperative Merging Assistant algorithm is a local control strategy managing each junction separately. The extent of the main carriageway was chosen to be sufficiently long to incorporate all the traffic phenomena occurring upstream and downstream of the merging location. The CoopMA sends the signal to decrease the speed of cooperative vehicles several kilometres before of the merging; therefore, the upstream stretch should be long enough to include this location. In addition, this link should be able to capture the eventual congestion that propagates upstream from the merging. Not all dynamics happen upstream, disruptions created by on-ramp vehicles could 


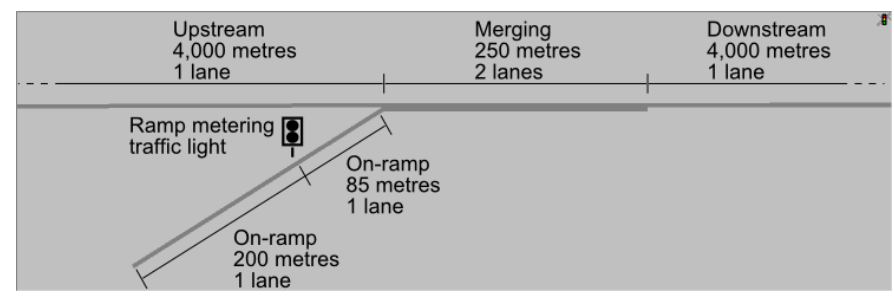

Fig. 3. Simulated infrastructure of the motorway junction using VISSIM (configured right-hand traffic). The junction is composed of: upstream, merging, downstream and on-ramp links with traffic light.

lead to flow break-down several kilometres downstream of the merging area. So the downstream stretch was chosen long enough to incorporate this phenomenon. To keep the simulation complexity at a minimum in order to have a clear evaluation of the effects of the CoopMA, a single lane motorway main carriageway has been simulated. Anyway, a single lane representation covers the essential system components, because the control policy communication and cooperation happen only on the near-side lane. In the case of a multi-lane motorway, additional lane changing behaviour should be considered and limited. Off-side lane changes, i.e. from the near-side (slow lane) towards the off-side (fast lane), can be allowed for any vehicle except the cooperative ones. This type of lane change will have the beneficial effect of increasing the gap available for merging, although the consequences on platoon formation should be evaluated carefully in simulations. Instead, to protect the gap created, near-side lane changes should be forbidden for all vehicles. These lane changing behaviours could be enforced applying one-side solid line on the pavement. For the same reason of having a clear evaluation of the CoopMA effects whilst keeping the simulation as simple as possible, the off-ramp that is usually present at motorway junctions has not been simulated. This allows reducing the complexity of weaving movements happening before the exit, and once again giving the possibility to focus on the CoopMA effects.

Based on these considerations, the infrastructure shown in Figure 3 has been simulated. The motorway stretch is composed of four links: an upstream link of $4 \mathrm{~km}, 1$ lane; a merging link of 250 metres, composed of two lanes, a main carriageway lane and an acceleration lane; a downstream link of $4 \mathrm{~km}, 1$ lane; and an on-ramp link of 285 metres, 1 lane, divided in two sections by the on-ramp traffic light situated 85 metres upstream of the start of the merging link. The length of the merging section, the on-ramp and the traffic light position are based on a standard English motorway junction [27].

Having presented the simulation characteristics, the measures of effectiveness (MoE) for evaluating the traffic performance are now introduced. Supposing the causes of breakdown are disruptions created by late-merging vehicles, a set of MoEs has been identified in order to evaluate if, by improving merging, the CoopMA can improve traffic performance for different on-ramp flows $q$. The four MoEs evaluated as performance criteria to answer the research questions Q1-Q4 are: (i) $\gamma_{q}$ occurrence of congestion [-] (proportion 0-1). The number $N_{c}$ of simulation runs with the creation of congestion divided by the total number $n$ of single run simulations for a specific scenario: $\gamma_{q}=N_{c} / n$. As previously mentioned, the same scenario is simulated with $n=30$ different random seeds; therefore this index can be interpreted as the rate of occurrence of congestion for on-ramp flow $q$. An event of congestion is classified as such if the average vehicle speed on a 10 metre section of main carriageway motorway is less than $40 \mathrm{~km} / \mathrm{h}$ for more than 10 seconds. This criterion has been chosen after several tests to identify clearly major disruptions in traffic flow, and the value of $40 \mathrm{~km} / \mathrm{h}$ is often used as a threshold for identifying congested-flow states [1].

(ii) $\tau_{q}$ proportion of time spent in congestion [-] (proportion 0-1). The proportion of time $T_{c}$ during which congestion is present in at least one 10 metre motorway cell, relative to the entire simulation time $t: \tau_{q}=T_{c} / t$. This index indicates the extension and severity of the congestion.

(iii) $\lambda_{q}$ proportion of late-merging vehicles [-] (proportion $0-1)$. Proportion of late merging vehicles $N_{l}$ on the total of merging vehicles $h$ when the simulation is not in congestion: $\lambda_{q}=N_{l} / h$. A vehicle is considered as late merging if it merges in the last 50 metres of the acceleration lane [28], i.e. after 200 metres from the start of the merging link. This index shows the proportion of vehicles that most likely are going to disrupt the traffic flow, because those vehicles are ready to accept smaller gaps and merges with slower speed.

(iv) $m_{q}$ merging position [metre, $0 \leq m_{q} \leq 250$ ]. The mean of the merging position of all merging vehicles when the simulation is not in congestion, relative at the beginning of the merging link. This index illustrates the position on the merging section at which vehicles are able to find a suitable gap for merging. If they find this gap at the beginning of the section, these vehicles are less likely to create disruption at the traffic flow.

Because multiple runs are used, the single run simulations MoEs are aggregated to obtain the multiple runs simulation MoEs. $\gamma_{q}$ is already an aggregate index, so it does not need further aggregation. $\tau_{q}$ is calculated as the average of all the single run simulations, and $\lambda_{q}$ and $m_{q}$ are the average of all individual values of the merging vehicles for all the single run simulations but only before congestion occurs. Only vehicles merging before congestion are considered for the calculation of $\lambda_{q}, m_{q}$, because, once congestion occurs, the merging process has a complete different dynamics, and it is no longer controlled by the CoopMA system, therefore these MoEs are no of interest. A warm-up period during which vehicles are partially present on the network has omitted.

The evaluation focuses on the ability of the CoopMA in preventing congestion, more than its performance once congestion has occurred. For this reason $\tau_{q}$, proportion of time spent in congestion, is used instead of Travel Time (TT). $\tau_{q}$ is a function of the moment when congestion starts, and it is less dependent on the spatio-temporal evolution of the queue than TT. $\tau_{q}$ shows more clear results on the performance during the comparison between scenarios without and with CoopMA; because the two scenarios present different 
evolution of queues after the breakdown that influence TT but not $\tau_{q}$.

The general associate hypothesis at the research questions Q.1-Q.4 is that the CoopMA is able to produce positive effects thanks to a facilitated merging process. To evaluate this general statement quantitatively, it is possible to state formal statistical hypotheses that should be tested comparing the values of MoEs for different scenarios (MoEs for the Reference scenario have been superscripted with the symbol $\mathrm{R}$ and for the CoopMA with the symbol $\mathrm{C}$ ). The four research hypotheses can be formally written as:

T.1 The hypothesis for Q.1 is: Cooperative Merging Assistant reduces the occurrence of congestion. Thus the test MoE is $\gamma_{q}$ occurrence of congestion (Reference vs. CoopMA)

$$
\begin{aligned}
& H_{0}: \gamma_{q}^{\mathrm{C}}=\gamma_{q}^{\mathrm{R}} \\
& H_{1}: \gamma_{q}^{\mathrm{C}}<\gamma_{q}^{\mathrm{R}}
\end{aligned}
$$

As already introduced, $\gamma_{q}$ is a binomial index for a single run simulation, i.e. each run can have values of 0 (congestion is not present) or 1 (congestion is present), therefore this hypothesis is evaluated with a test for difference in two population proportions.

T.2 The hypothesis for Q.2 is: Cooperative Merging Assistant reduces the proportion of time spent in congestion. Thus the test $\mathrm{MoE}$ is $\tau_{q}$ proportion of time spent in congestion (Reference vs. CoopMA)

$$
\begin{aligned}
& H_{0}: \tau_{q}^{\mathrm{C}}=\tau_{q}^{\mathrm{R}} \\
& H_{1}: \tau_{q}^{\mathrm{C}}<\tau_{q}^{\mathrm{R}}
\end{aligned}
$$

This hypothesis is evaluated with a paired $t$-test where single run simulations with the same random number for the two different scenarios are handled as a pair.

T.3 The hypothesis for Q.3 is: Cooperative Merging Assistant reduces the proportion of vehicles that merge late. Thus the test MoE is $\lambda_{q}$ proportion of late-merging vehicles (Reference vs. CoopMA)

$$
\begin{aligned}
& H_{0}: \lambda_{q}^{\mathrm{C}}=\lambda_{q}^{\mathrm{R}} \\
& H_{1}: \lambda_{q}^{\mathrm{C}}<\lambda_{q}^{\mathrm{R}}
\end{aligned}
$$

As in T.1, this hypothesis is evaluated with a test for difference in two population proportions, but in this case the sample size consists of all merging vehicles. Each vehicle is considered as a binomial variable that could be a latemerging vehicle or not.

T.4 The hypothesis for Q.4 is: vehicles can merge earlier under Cooperative Merging Assistant than they could without it. Thus the test MoE is $m_{q}$ merging position (Reference vs. CoopMA)

$$
\begin{aligned}
& H_{0}: m_{q}^{\mathrm{C}}=m_{q}^{\mathrm{R}} \\
& H_{1}: m_{q}^{\mathrm{C}}<m_{q}^{\mathrm{R}}
\end{aligned}
$$

This hypothesis is evaluated with a standard $t$-test for two populations with unknown variance where the position of each merging vehicle is considered a sample for each scenario.

The four tests are based on the comparison between the
Reference and CoopMA scenario, and not between with and without CoopMA because the latter is expected to perform worse in all cases than the unmanaged situation. An increase in the occurrence of congestion, time spent in congestion, late-merging vehicles and a more advanced merging position are expected in comparison with both Reference and CoopMA cases. This is because, when vehicles are released by a traffic light as in the RM system, the merging manoeuvre may become more difficult, and onramp vehicles, in particular if released in platoons, can create disruptions stronger than in the uncontrolled scenario. As previously mentioned, this scenario aims to compare the disruptions caused to the traffic flow by the presence of a traffic light without facilitated merging.

For the simulations the combination of MATLAB version R 2012b and the VISSIM version 5.40 have been adopted. VISSIM uses a psycho-physical car following model based on [29] and a lane-changing/merging model incorporating courtesy lane-changing and courtesy yielding behaviour. This tool has been used in recent evaluations of cooperative merging control strategies [22], [23].

The default parameters of VISSIM have been proved to reproduce the significant traffic phenomena, as will be shown in Section IV. Congestion develops on the main carriageway in proximity of the merge, and then it propagates upstream with a speed of about $-18 \mathrm{~km} / \mathrm{h}$, in agreement with empirical observation [1]. Break-down is often simulated as occurring several hundred metres downstream to the merging section, reproducing correctly the relaxation phenomenon and the boomerang effect [7], [30]. Also the merging process is correctly represented; the positions where on-ramp vehicles are able to move on the main carriageway are in agreement with empirical observations, and the important courtesy yielding manoeuvre is incorporated too [28], [31].

\section{RESULTS AND DISCUSSION}

In this section, before discussing and comparing the simulation results, the spatio-temporal diagrams of significant runs and the headway distributions are presented in order to have a qualitative understanding of the simulation behaviour and the different types of congestion created.

Figure 4 shows examples of queue formation and propagation for increasing on-ramp flows using spatio-temporal diagrams of the vehicle speed on the main carriageway in the case of the Reference scenario. The abscissa indicates time in seconds for the 30 minute simulated time horizon, and the ordinate indicates the main carriageway motorway location, from $4 \mathrm{~km}$ upstream of the merging area to $4 \mathrm{~km}$ downstream. The start and end of the 250 metre merging section are indicated with black solid lines. Figure 4 (a) (on-ramp flow $450 \mathrm{veh} / \mathrm{h}$, run 7) shows an example of an extended and severe perturbation. The disruption to traffic flow is not sufficiently strong to lead to a unrecoverable break-down, and after about 500 seconds the simulation returns to a free-flow state. Instead, a different simulation run with the same on-ramp flow leads to unrecoverable transition from free-flow to congested-flow, Figure 4 (b) 

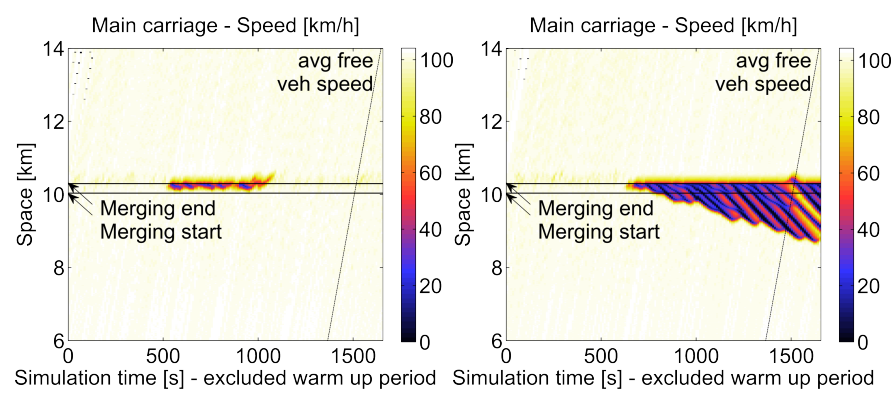

(a)

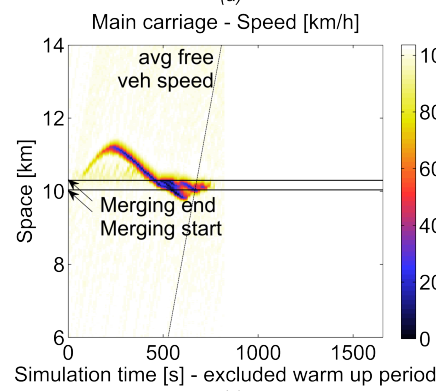

(c) (b)

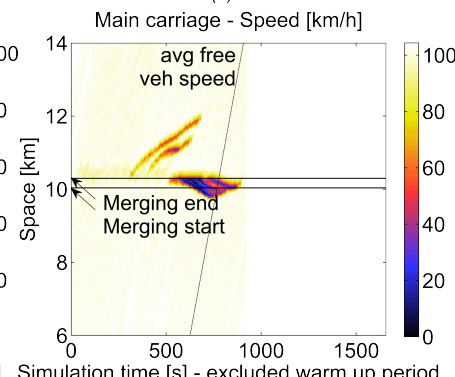

(d)

Fig. 4. Spatio-temporal diagrams of different congestion formation for the Reference scenario. (a) severe congestion (on-ramp flow $450 \mathrm{veh} / \mathrm{h}$, run 7). (b) break-down and shock wave formation (on-ramp flow $450 \mathrm{veh} / \mathrm{h}$, run 15). (c) boomerang effect (on-ramp flow $800 \mathrm{veh} / \mathrm{h}$, run 1). (d) example of complex behaviour (on-ramp flow $800 \mathrm{veh} / \mathrm{h}$, run 5).

(on-ramp flow $450 \mathrm{veh} / \mathrm{h}$, run 15). In this case the initial disruptions at around 700 seconds create a flow breakdown with consequent upstream propagation of shock waves. Instead, Figure 4 (c) (on-ramp flow $800 \mathrm{veh} / \mathrm{h}$, run 1) shows a different example of a break-down, and in this case the boomerang phenomenon is clearly visible. Perturbations generated at the merging location increase in magnitude while are propagating downstream, until they create a break-down about $1 \mathrm{~km}$ from the junction, in agreement with stability theory and empirical observations [7]. Then, a shock wave is generated and starts moving upstream, until it reaches the merging section, the location where the first disruption took place. As visible, this simulation run has been interrupted before the end of the 30 minutes horizon, at 800 seconds. This is because under strong congestion, the simulation reproduces accurately the occurrence of break-down and the shock wave propagation, but not the behaviour of onramp vehicles once a shock wave has reached the merging location. In some cases, on-ramp vehicles are completely stationary, i.e. zero speed, and are not able to merge the motorway, while mean carriageway vehicles start travelling at free speed. This behaviour is not representative of the real situation; therefore, the simulation is stopped and classified as being in a congested state from the moment of the breakdown onwards. The final figure, Figure 4 (d) (on-ramp flow $800 \mathrm{veh} / \mathrm{h}$, run 5), presents an example of complex behaviour reproduced by the simulation. Two perturbations created near the merging section propagate downstream but do not lead to break down because the upstream flow has been reduced by a third perturbation formed at the merging location. The latest disruption creates a not-recoverable congestion at 550 seconds, leading to a total blockage of the on-ramp and
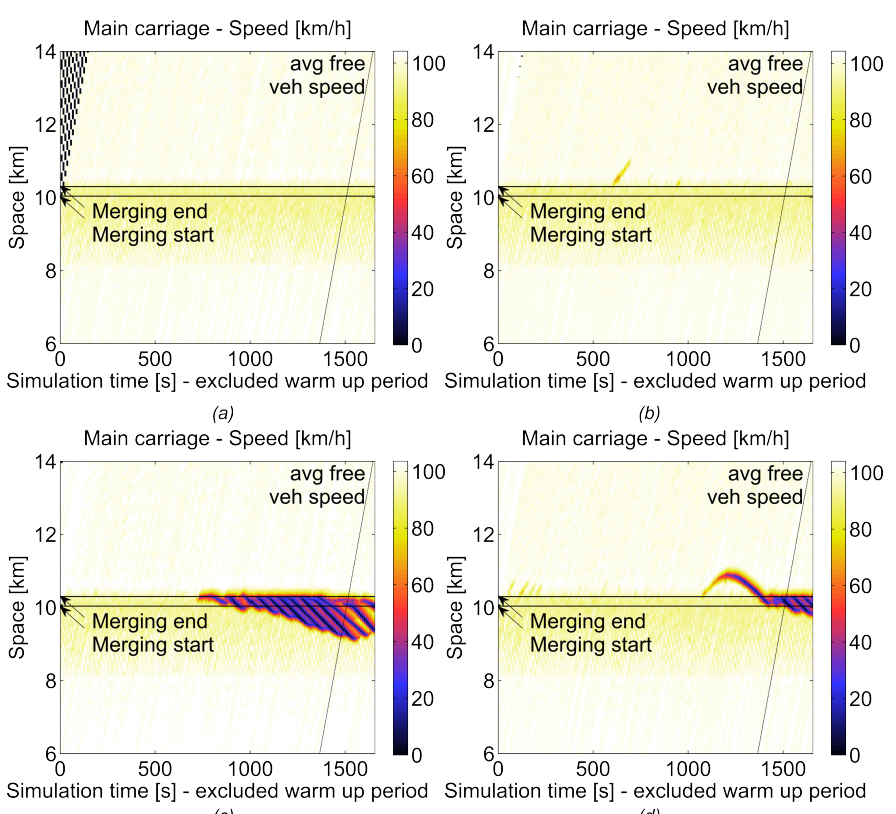

(c)

Fig. 5. Spatio-temporal diagrams of different congestion formation for the Cooperative Merging Assistant scenario. (a) complete absence of congestion and visible cooperative area (on-ramp flow $200 \mathrm{veh} / \mathrm{h}$, run 1). (b) small perturbation not classified as congestion (on-ramp flow $650 \mathrm{veh} / \mathrm{h}$, run 18). (c) break-down and shock wave formation (on-ramp flow $650 \mathrm{veh} / \mathrm{h}$, run 11). (d) boomerang effect (on-ramp flow $750 \mathrm{veh} / \mathrm{h}$, run 23 ).

consequent stop of the simulation run.

Looking at the variability present among the different runs, it is clear that a range of traffic flow phenomena are reproduced. Furthermore it is interesting to notice that the same scenario simulated with different random seeds can bring to completely different results, eg Figure $4(a)$ and $(b)$. This supports the idea that facilitating the merging process could lead to the prevention of the single disruption that triggered the non-recoverable transition from free-flow to congested flow, and it is within this logic that the CoopMA operates.

The qualitative traffic flow phenomena in the Without CoopMA scenario are similar to the one of the Reference scenario; therefore, no spatio-temporal diagrams are presented. However, the impacts on the traffic performance caused by the presence of a traffic light and the release of on-ramp platoons without main carriageway cooperation are discussed in the following.

The final simulated scenario analyses the traffic performance under the control of the CoopMA. The traffic behaviour for this scenario is shown in Figure 5, where the spatio-temporal diagrams of the main carriageway speed for some relevant runs are presented. In contrast with the Reference scenario, from $\mathrm{km} 8$ to $\mathrm{km} \mathrm{10,} \mathrm{the} \mathrm{area} \mathrm{is} \mathrm{visible}$ where the cooperative vehicles decrease their speed and start the formation of 10-vehicle platoons. Figure 5 (a) (on-ramp flow $200 \mathrm{veh} / \mathrm{h}$, run 1) is an example of a run where the use of CoopMA is able to remove all perturbations in the merging area. The only disruptions to the traffic flow are the ones introduced by the CoopMA strategy itself, but, as is visible, they provide a smooth transition from state 


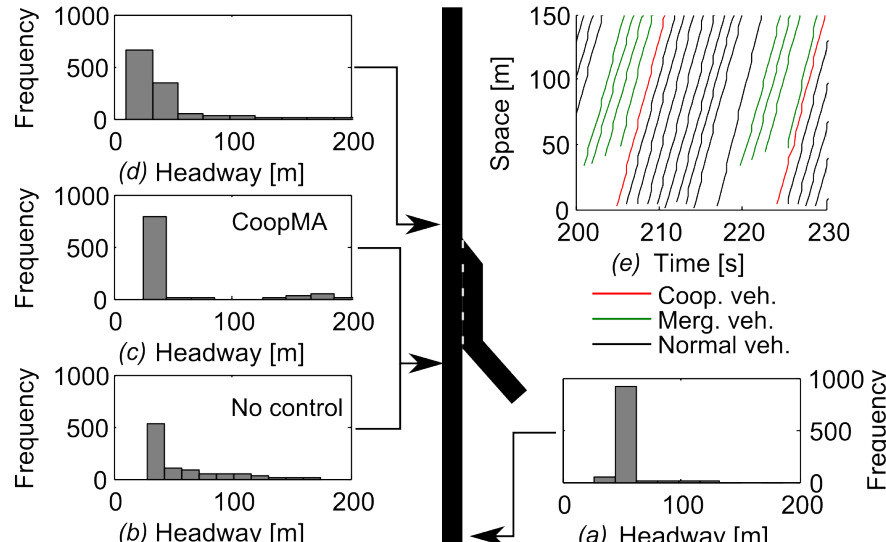

Fig. 6. Headway distributions in different sections of the infrastructure. (a) generation section. (b) upstream the merging section in the uncontrolled scenarios. (c) upstream the merging section when CoopMA is applied. (d) downstream the merging section. $(d)$ vehicle trajectories in the merging section, main carriageway lane.

$A$, natural traffic flow state, to state $C$, cooperative state with platoons and gaps, without generating shock waves itself. In Figure 5 (b) (on-ramp flow $650 \mathrm{veh} / \mathrm{h}$, run 18) a small perturbation, not classified as congestion, is visible at 600 seconds that, although propagating downstream, does not lead to break-down. Instead, Figure 5 (c) (on-ramp flow $650 \mathrm{veh} / \mathrm{h}$, run 11) and (d) (on-ramp flow $750 \mathrm{veh} / \mathrm{h}$, run 23) show two examples of non-recoverable congestion with two different evolutions. In the first case traffic breaks down at the merging location, and then shock waves are created, similar to Figure $4(b)$. In the second case, after several perturbations without consequence in the first 200 seconds, flow breaks down at 1200 seconds reproducing the boomerang phenomenon, in analogy with Figure 4 (c).

Figure 6 shows the headway distributions in different sections of the infrastructure, as well as a sample of the vehicle trajectories in the merging section. In all scenarios, vehicles are generated with a average headway of 50 metre with some random variations as previously described, Figure $6(a)$. Then, when they reach the merging section in the uncontrolled and Without CoopMA scenarios, the vehicles are naturally grouped in random platoons due to the presence of vehicles with lower desired speed [28], as shown by Figure $6(b)$. On the other hand, when the CoopMA is applied, two distinct groups are present, Figure 6 (c). Compacted vehicles with small headways, and cooperative vehicles with in front large headways used for merging. Figure $6(e)$ shows how these gaps are efficiently used by onramp vehicles, which find available space exactly in the moment of the merging. The absence of perturbations is visible from the nearly straight trajectories. Finally, downstream of the merging section, in all cases, vehicles travel with smaller average headways, 40 metre in this example Figure $6(d)$, due to the increased total flow.

Figure 7 shows the trends for the MoEs presented in Section III. The abscissa for all figures indicates the onramp flow, while the ordinate represents the value of various
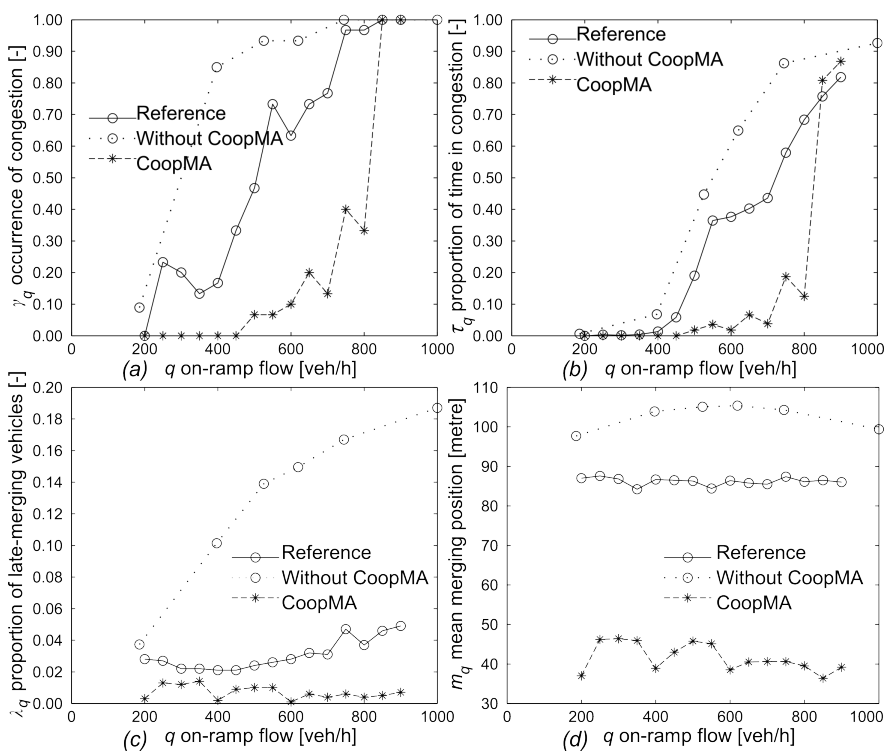

Fig. 7. Results and comparison for the scenarios. (a) $\gamma_{q}$ occurrence of congestion, (b) $\tau_{q}$ proportion of time spent in congestion, (c) $\lambda_{q}$ proportion of late-merging vehicles and $(d) m_{q}$ mean of the merging position.

indexes for the different scenarios. The following is a discussion for each index.

The index occurrence of congestion $\gamma_{q}$ - Figure 7 (a), can be interpreted as the rate of breakdown at flow $q$. For all scenarios $\gamma_{q}$ increases with the increase of on-ramp flow, and it reaches $100 \%$ for $q$ higher than $850 \mathrm{veh} / \mathrm{h}$. For on-ramp flows less than this value, in comparison with the uncontrolled scenario, the release of platoons without main carriageway collaboration increases the occurrence of congestion due to the more intense perturbations created by merging vehicles. Stronger disruptions are caused by the increasing difficulty in the merging manoeuvre when vehicles are released by an on-ramp traffic light [32], and by the merging of on-ramp platoons leading to an increased number of late-merging vehicles, as shown by Figure 7 (c). On the other hand, the use of CoopMA greatly reduces the occurrence of congestion thanks to the creation of suitable gaps for merging. This result is obtained by a reduction in late-merging vehicles, Figure 7 (c), and so, a decrease of disruptions that could lead to congestion.

The index proportion of time spent in congestion $\tau_{q}$ Figure 7 (b) shows similar trends to $\gamma_{q}$ for all on-ramp flows, showing that CoopMA can postpone the creation of congestion and so reduce the proportion of time spent in congestion. The reason for this behaviour is once again the reduction of late-merging vehicles thanks to the facilitated merging process.

As in the case of $\gamma_{q}$ and $\tau_{q}$, the trends for the proportion of late-merging vehicles $\lambda_{q}$ - Figure 7 (c), show clear results on the effectiveness of the CoopMA algorithm. The use of CoopMA reduces the number of late-merging vehicles at each flow level in comparison with the other scenarios. In the Without CoopMA scenario, $\lambda_{q}$ increases progressively, showing that on-ramp vehicles have difficulties in finding suitable gaps, in particular when released in platoons. A 
TABLE I

P-VALUES FOR THE STATISTICAL TESTS

\begin{tabular}{|c|c|c|c|c|}
\hline $\begin{array}{c}\text { On-ramp flow } \\
q[\mathrm{veh} / \mathrm{h}]\end{array}$ & $\begin{array}{l}\text { T.1 } \\
\gamma_{q}\end{array}$ & $\begin{array}{l}\mathrm{T} .2 \\
\tau_{q}\end{array}$ & $\begin{array}{l}\text { T.3 } \\
\lambda_{q}\end{array}$ & $\begin{array}{l}\text { T. } 4 \\
m_{q}\end{array}$ \\
\hline 200 & 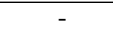 & - & $<10^{-16}$ & $<10^{-16}$ \\
\hline 250 & 0.008 & $<10^{-16}$ & $2 \times 10^{-08}$ & $<10^{-16}$ \\
\hline 300 & 0.015 & 0.013 & 0.005 & $<10^{-16}$ \\
\hline 350 & 0.061 & 0.067 & 0.237 & $<10^{-16}$ \\
\hline 400 & 0.031 & 0.026 & $<10^{-16}$ & $<10^{-16}$ \\
\hline 450 & 0.001 & 0.001 & $6 \times 10^{-08}$ & $<10^{-16}$ \\
\hline 500 & 0.001 & 0.002 & $1 \times 10^{-09}$ & $<10^{-16}$ \\
\hline 550 & $2 \times 10^{-07}$ & $9 \times 10^{-05}$ & $<10^{-16}$ & $<10^{-16}$ \\
\hline 600 & $9 \times 10^{-06}$ & $4 \times 10^{-06}$ & $<10^{-16}$ & $<10^{-16}$ \\
\hline 650 & $3 \times 10^{-05}$ & $8 \times 10^{-05}$ & $<10^{-16}$ & $<10^{-16}$ \\
\hline 700 & $1 \times 10^{-07}$ & $2 \times 10^{-07}$ & $<10^{-16}$ & $<10^{-16}$ \\
\hline 750 & $3 \times 10^{-07}$ & $7 \times 10^{-06}$ & $<10^{-16}$ & $<10^{-16}$ \\
\hline 800 & $3 \times 10^{-06}$ & $1 \times 10^{-07}$ & $<10^{-16}$ & $<10^{-16}$ \\
\hline 850 & 0.638 & 0.774 & $<10^{-16}$ & $<10^{-16}$ \\
\hline 900 & 0.745 & 0.909 & $3 \times 10^{-12}$ & $<10^{-16}$ \\
\hline
\end{tabular}

small increase of $\lambda_{q}$ is visible for the uncontrolled scenario proving that on-ramp vehicles have difficulties in finding suitable gaps in dense traffic; meanwhile, in the CoopMA scenario it remains almost constant, showing the ability of this innovative system to keep $\lambda_{q}$ low even for high on-ramp flows. Therefore, assuming that late-merging vehicles are the most responsible for creating disruptions that could lead to congestion, this index shows why the system is able to reduce the occurrence and intensity of it.

As expected, providing suitable gaps for merging, CoopMA is able to decrease considerably the average merging position of on-ramp vehicles $m_{q}$ - Figure $7(d)$. While in this scenario almost the totality of the on-ramp vehicles is able to merge at the beginning of the on-ramp, in the case of uncontrolled and without main carriageway cooperation, they merge around the middle of the section, in agreement with empirical observations [28]. The average merging position in the Without CoopMA scenario is the most downstream because the vehicle trajectories are disturbed by the presence of the traffic light, and no assistance is given from main carriageway vehicles as in the case of CoopMA.

The graphical investigation of the MoE shows clear results of the positive effects of the CoopMA on traffic flow. This conclusion can be also supported in a more formal way. The research questions on the effectiveness of CoopMA, Q.1-Q.4, can be answered using the statistical test, T.1-T.4, presented in Section III. Table I reports the $p$-values of the results of the four hypotheses. It is convenient to remember that a widely used significance level for rejecting the null hypothesis is $p$-value $<0.05$ [33]. Therefore, for how T.1-T.4 have been designed, each test that has a $p$-values less than 0.05 can be interpreted as having enough statistical evidence to support the positive effect of the CoopMA on traffic flow. Analysing the results in Table I, the qualitative conclusions drawn from the indexes trends in Figure 7 are confirmed by quantitative indications.

Few considerations should be made on the cases where the $p$-values are greater than 0.05 , i.e. there is no enough statistical evidence to support the positive effect of the CoopMA.
The first case is for the indexes occurrence of congestion $\gamma_{q}$, proportion of time spent in congestion $\tau_{q}$ and proportion of late-merging vehicles $\lambda_{q}$ for flow $q=350 \mathrm{veh} / \mathrm{h}$. As it is also visible from the MoE trends in Figure 7, for flows lower than this value, the disruptions to the traffic are limited. This because, the total flow is well under capacity, and the junction can be considered mostly in free-flow state. For this reason, applying the CoopMA, although facilitate the merging process, does not bring evident improvement to the traffic condition, which already presents few events of congestion. The second case is for the indexes $\gamma_{q}$ and $\tau_{q}$ for $q \geq 850 \mathrm{veh} / \mathrm{h}$. In this case, the traffic on the motorway has an elevated density, and few empty spaces are left to be rearranged by the CoopMA system. As expected, in this situation, there is almost no scope for the traffic management system to improve traffic performance, and the only possible control option could be to reduce the demand.

In conclusion, given the graphical interpretations and the statistical results, there is sufficient evidence to answer all research questions positively and to support the capability of the Cooperative Merging Assistant system in improving traffic performance at motorway junctions.

\section{CONCLUSIONS}

This paper evaluated the effects of the Cooperative Merging Assistant control strategy on the traffic flow. The proposed cooperative system operates by modifying the distribution of gaps between vehicles on the main carriageway, but without reducing flow and slowing some vehicles only slightly. In particular, it has the effect of collecting several relatively small partial headways into a single longer one that can be used to facilitate merging. This approach cannot increase the capacity of the main carriageway, which has to be sufficient for the total inflow if merging is to be possible at all. However, by facilitating the merging process itself, it increases the flows that can be accommodated without transient caused by merging precipitating flow breakdown.

Based on the simulation results and the statistical tests, the hypothesis that the Cooperative Merging Assistant could improve the traffic performance has been confirmed. CoopMA, by providing suitable gaps for merging, can greatly reduce the number of late-merging vehicles, thought to be the prime cause of flow breakdown at merging. This innovative strategy, reducing merging disruptions, is able to decrease the occurrence of congestion for a wide range of on-ramp flows and to reduce the time spent in congestion as well.

Having confirmed the effectiveness of the CoopMA system, a further consideration should be made on how to use this reduction of congestion. It can be used in two ways by a motorway operator. Assuming the stochastic nature of breakdown [4], the capacity of the motorway in proximity of a junction can be defined as the flow associated with a certain rate of breakdown, evaluated with the index $\gamma_{q}$. Therefore, the use of CoopMA, reducing this value, is actually increasing the capacity; and so, without physical intervention, the operator can increase the motorway throughput. A second possibility is to increase the reliability 
of the service provided. Assuming that a junction is already controlled by a traditional RM system, the CoopMA could be used to facilitate the merging. With this configuration, instead of increasing the target on-ramp flow to match the increased capacity, the operator can decide to maintain the same target flow currently used by traditional RM; and, thanks to the use of CoopMA, decrease the occurrence of congestion. This means that the drivers will experience a more reliable service, undergoing fewer events of recurrent congestion.

To have a complete evaluation of the CoopMA system, a wider range of scenarios needs to be investigated, e.g. multiple lanes, different penetration rates, different main carriageway demands. All these aspects will be assessed in future work.

\section{ACKNOWLEDGEMENTS}

The authors are grateful to the EC FP7 NEARCTIS project for funding this research.

\section{REFERENCES}

[1] B. S. Kerner, The physics of traffic: empirical freeway pattern features, engineering applications, and theory, ser. Understanding complex systems. Berlin: Springer, 2004.

[2] M. J. Lighthill and G. B. Whitham, "On kinematic waves. II. A theory of traffic flow on long crowded roads," Proceedings of Royal Society, vol. 229A, no. 1178, pp. 317-345, 1955.

[3] I. Prigogine and R. Herman, Kinetic theory of vehicular traffic. American Elsevier Pub. Co, 1971.

[4] W. Brilon, J. Geistefeldt, and H. Zurlinden, "Implementing the concept of reliability for highway capacity analysis," Transportation Research Record, no. 2027, pp. 1-8, 2007.

[5] R. L. Bertini and S. Malik, "Observed dynamic traffic features on freeway section with merges and diverges," Transportation Research Record, no. 1867, pp. 25-35, 2004.

[6] A. Kotsialos, M. Papageorgiou, J. Hayden, R. Higginson, K. McCabe, and N. Rayman, "Discrete release rate impact on ramp metering performance," IEE Proceedings: Intelligent Transport Systems, vol. 153, no. 1, pp. 85-96, 2006.

[7] M. J. Cassidy and R. L. Bertini, "Some traffic features at freeway bottlenecks," Transportation Research Part B: Methodological, vol. 33, no. 1 , pp. 25-42, 1999.

[8] L. Leclercq, J. A. Laval, and N. Chiabaut, "Capacity drops at merges: An endogenous model," Transportation Research Part B: Methodological, vol. 45, no. 9, pp. 1302-1313, 2011.

[9] M. Papageorgiou and A. Kotsialos, "Freeway Ramp Metering: an overview," IEEE Transactions on Intelligent Transportation Systems, vol. 3, no. 4, pp. 271-281, 2002.

[10] D. P. Masher, Guidelines for design and operation of ramp control systems. Stanford Research Institute National Cooperative Highway Research Program. Transportation Research Board, 1975.

[11] M. Papageorgiou, H. Hadj-Salem, and J. M. Blosseville, "ALINEA: a local feedback control law for on-ramp metering," Transportation Research Record, no. 1320, pp. p. 58-64, 1991.

[12] B. S. Kerner, "On-ramp metering based on three-phase traffic theory Part II," Traffic Engineering and Control, vol. 48, no. 2, pp. 68; 8-75, 2007.

[13] T. Bellemans, B. D. Schutter, and B. D. Moor, "Model predictive control for ramp metering of motorway traffic: A case study," Control Engineering Practice, vol. 14, no. 7, pp. 757-767, 2006.

[14] R. Scarinci and B. G. Heydecker, "Control concepts for facilitating motorway on-ramp merging using intelligent vehicles," Transport Reviews, vol. 34, no. 6, pp. 775-797, 2014.

[15] P. Kachroo and Z. Li, "Vehicle merging control design for an automated highway system," in IEEE Conference on Intelligent Transportation Systems, ITSC, 1997, pp. 224-229.

[16] B. Ran, S. Leight, and B. Chang, "A microscopic simulation model for merging control on a dedicated-lane automated highway system," Transportation Research Part C: Emerging Technologies, vol. 7, no. 6, pp. 369-388, 1999.
[17] Q. Xu and R. Sengupta, "Simulation, analysis, and comparison of ACC and CACC in highway merging control," in Intelligent Vehicles Symposium IEEE proceedings, 2003, pp. 237-242.

[18] L. C. Davis, "Effect of adaptive cruise control systems on mixed traffic flow near an on-ramp," Physica A: Statistical Mechanics and its Applications, vol. 379, no. 1, pp. 274-290, 2007.

[19] W. Daamen, B. van Arem, and I. Bouma, "Microscopic dynamic traffic management: simulation of two typical situations," in 14th IEEE International Intelligent Transportation Systems Conference, ITSC, 2011, pp. 1898-1903.

[20] Z. Wang, L. Kulik, and K. Ramamohanarao, "Proactive traffic merging strategies for sensor-enabled cars," in VANET'07: Fourth ACM International Workshop on Vehicular Ad Hoc Networks, 2007, pp. 39-48.

[21] R. Pueboobpaphan, F. Liu, and B. van Arem, "The impacts of a communication based merging assistant on traffic flows of manual and equipped vehicles at an on-ramp using traffic flow simulation," in 13th International IEEE Conference on Intelligent Transportation Systems, ITSC, 2010, pp. 1468-1473.

[22] H. Park, C. S. Bhamidipati, and B. L. Smith, "Development and evaluation of enhanced intellidrive-enabled lane changing advisory algorithm to address freeway merge conflict," Transportation Research Record, no. 2243, pp. 146-157, 2011.

[23] D. Marinescu, J. Curn, M. Bouroche, and V. Cahill, "On-ramp traffic merging using cooperative intelligent vehicles: A slot-based approach," in 15th International IEEE Conference on Intelligent Transportation Systems, ITSC, 2012, pp. 900-906.

[24] M. Papageorgiou and I. Papamichail, "Overview of traffic signal operation policies for ramp metering," Transportation Research Record, no. 2047, pp. 28-36, 2008.

[25] R. Scarinci, "Managing traffic at motorway junctions: a ramp metering development using intelligent vehicles," Ph.D. dissertation, University College London UCL, 2014.

[26] R. Scarinci, A. Hegyi, and B. G. Heydecker, "Cooperative Ramp Metering A Study of the Practicality of a Ramp Metering Development Using Intelligent Vehicles," in 45th Annual UTSG Universities' Transport Study Group Conference, 2013.

[27] DfT, Design manual for roads and bridges, Highways Agency, Ed. Great Britain: Department of Transport, 2011.

[28] W. Daamen, M. Loot, and S. P. Hoogendoorn, "Empirical analysis of merging behavior at freeway on-ramp," Transportation Research Record, no. 2188, pp. 108-118, 2010.

[29] R. Wiedemann, "Simulation des strassenverkehrsflusses," Institute for Traffic Engineering, University of Karlsruhe, Tech. Rep., 1974.

[30] S. Kim and B. Coifman, "Driver relaxation impacts on bottleneck activation, capacity, and the fundamental relationship," Transportation Research Part C: Emerging Technologies, 2013.

[31] F. Marczak, W. Daamen, and C. Buisson, "Key Variables of Merging Behaviour: Empirical Comparison between Two Sites and Assessment of Gap Acceptance Theory," Procedia-Social and Behavioral Sciences, vol. 80, pp. 678-697, 2013.

[32] P. Zheng and M. McDonald, "Evaluation of effects of ramp metering on merging operations," Transportation Research Record, no. 2012, pp. 105-112, 2007.

[33] D. C. Montgomery and G. C. Runger, Applied Statistics and Probability for Engineers. John Wiley and Sons, 2010.

Riccardo Scarinci received the MSc degree (2008) in environmental engineering from the Politecnico di Milano, Italy, and $\mathrm{PhD}$ in transport studies from UCL (2014). Currently he is a post-doctoral researcher at EPFL, Lausanne, working on simulation and modelling of transport systems.

Benjamin Heydecker received BA mathematics from Cambridge University (1978) and $\mathrm{PhD}$ in transport studies from UCL (1983). Following post-doctoral research at ITS, University of Leeds, he became Lecturer at UCL (1985) and Professor of Transport Studies (1999). Prof. Heydecker applies mathematical and statistical analysis and modelling to transport systems and their usage, with special interest in the opportunities furnished by emerging ICT.

Andreas Hegyi received the MSc degree (1998) and PhD degree (2004) from TU Delft. He performed post-doctoral research at TU Delft en Ghent University, and currently he is Assistant Professor at TU Delft. His research interests include control of freeway and urban traffic networks, cooperative systems and traffic state estimation. Dr. Hegyi is Associate Editor of IEEE Transactions on Intelligent Transportation Systems, member of IEEE, IEEE ITSS and the Editorial Board of Transportation Research Part C, and organizer of the IEEE ITSC 2013 conference. 